\title{
NEW DESIGNS FOR SUBMILLIMETRIC PRESS-FITTING
}

\author{
Ludovic Charvier, Fabien Bourgeois, Jacques Jacot
}

Laboratoire de Production Microtechnique, EPFL

CH-1015 Lausanne, Switzerland

ludovic.charvier@epfl.ch

\section{Grégoire Genolet, Hubert Lorenz}

Mimotec SA, Blancherie 61

CH-1950 Sion, Switzerland

info@mimotec.ch

\begin{abstract}
Press-fitting is a very common assembly process used in many fields, especially in the watchmaking industry. It consists of introducing a shaft in a hole (also called receptor) with a certain value of tightening (or interference). However, this process is not straightforward and it is often observed that some assemblies do not manage to hold out the time and function they are meant to. In order to solve this problem, influent parameters had been searched and are now studied. The shape of the receptor has appeared as a critical one. Indeed, new machining processes have been developed this last decade and we are now able to make new designs which could cope perfectly with this application. The article will deal with the influence of the two main parameters involved in a press-fitting assembly: the maximal press-fitting force, measured axially; and the resistive torque exercised between the shaft and the receptor. The main purpose will be to find which kind of design allows the contact to become less stiff, which means less internal constraints. Thus, the tolerances, especially those of the receptor (they are less well controlled than those of the shaft) could be enlarged and this would be a great improvement for the industrial world.
\end{abstract}

\section{Introduction}

Press-fitting is a joining process which is very simple to set up. Nevertheless, when the dimensions are smaller than one millimetre, everything becomes much more complicated to control. The main difficulty is that the tolerances demanded on the shaft and on the hole are extremely thin (about $1 \mu \mathrm{m}$ ). By consequence, the manufacturers are no longer able to cope with the requirements. Bourgeois showed that the assembly force is proportional to the interference and the stiffness of the receptor. The main idea of this study is so to reduce the stiffness of the design that the

Please use the following format when citing this chapter:

Charvier, L., Bourgeois, F., Jacot, J., Genolet, G., Lorenz, H., 2008, in IFIP International Federation for Information Processing, Volume 260, Micro-Assembly Technologies and Applications, eds. Ratchev, S., Koelemeijer, S., (Boston: Springer), pp. 67-74. 
tolerances acceptable for the assembly could be enlarged. This idea is illustrated in Figure 1.

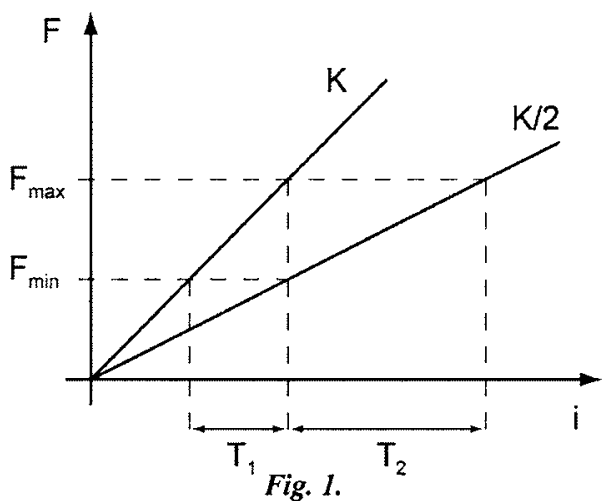

Evolution of the interference range when the stiffness of the contact is divided by two

Indeed, if we divide the stiffness of the contact ( $\mathrm{K}$ on the picture) by a factor two, we obtain a range for the tolerance $T$ which is about two times larger, for the same $F_{\min }$ and $F_{\max }$. These two forces represent the maximum and minimum axial forces the contact has to support.

We also observe that the interference range is moved, which may raise problems for some applications. However, we better understand now the interest in trying to reduce as much as possible the stiffness of the assembly.

Moreover, another point has to be taken into consideration. Press-fitting can be used so that the shaft resists to an axial force but in some other cases, it also has to resist to a minimum axial torque. The consequence is that the contact surface has to be increased while the resistive torque directly depends on this factor. Here is the formula of the resistive torque given by the Lamé-Clapeyron's model [1] which explains that dependence:

$$
M=F \cdot \frac{D}{2}=\frac{\pi}{4} \mu \cdot i \cdot D \cdot E \cdot L
$$

Fig. 2.

Resistive torque formula given by Lamé-Clapeyron's model

where $M$ is the resistive torque, $\mu$ the friction coefficient, $i$ the interference, $D$ the shaft diameter, $\mathrm{E}$ the Young modulus and $\mathrm{L}$ the length of contact.

Here $\pi$.D.L represents the contact surface for a proportion of contact of 1 but which can be modified as we will present later on. 


\section{Ideas of the experiment}

The main idea of this series of experiments is to reduce the stiffness of the contact in keeping a good contact surface for the resistive torque. For that, we decided to design new receptor shapes while shaft fabrication is better controlled. We can know the diameter of a shaft with a tolerance of $\pm 1 \mu \mathrm{m}$, which is completely impossible for a cylindrical hole. This is due to manufacturing processes and measuring tools which have about the same precision as the tolerance we want to obtain.

First of all, we have chosen to make our new receptors with a LIGA process. Thus, we have almost no constraints for the shape we want to design. Moreover, we can obtain a thickness up to $400 \mu \mathrm{m}$ which is typically the interesting range for our application.

Concerning the shape, we have tried to focus on the fact that the matter is less constrained when it works in traction/compression instead of flexion. With this approach, the receptor material would remain in its elastic field and the stiffness of the contact would be reduced. In order to show this influence, we decided to make designs with and without flexion oblongs. The following figures show this aspect:

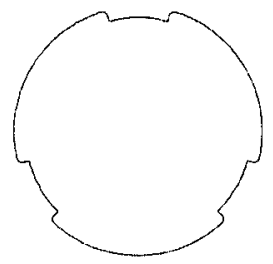

Fig. 3.

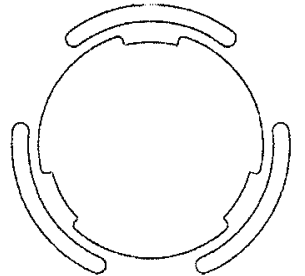

Fig. 4.

Designs with and without flexion oblongs

Another point which had been considered as primordial was to study the influence of the contact surface. For this, we decided to experiment with designs with different proportions of surface in contact with the pin. For instance, in Figures 5 and 6 we can observe a proportion of a half and of three thirds.
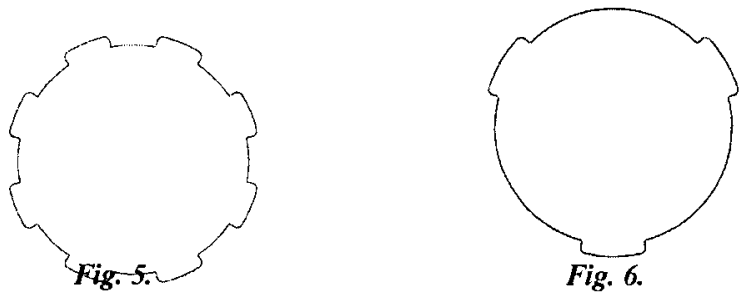

Designs with different proportions in contact with the pin 
And at least another parameter we would have studied was the micro oxydations. Indeed, in a previous study lead by Fabien Bourgeois [2], we noticed that some of these phenomena could appear during the press-fitting. We decided to test some shapes, with cannels, the goal of which would be to evacuate these microweldings. The following figures show the designs we developed to investigate this field:

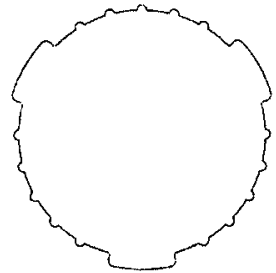

Fig. 7.

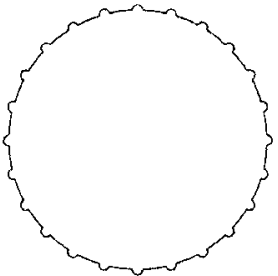

Fig. 8.

Designs with channels to evacuate the eventual microweldings

\section{Experimentation description}

The experiments are made on a Promess press whose maximal delivered force is $1000 \mathrm{~N}$. In this part, we will study the maximal press-fitting force obtained by each design and also the resistive torque associated with each particular shape.

Figure 9 shows the "posage" used to align the shaft with the design to test. This device is also used to determine the maximal press-fitting force.

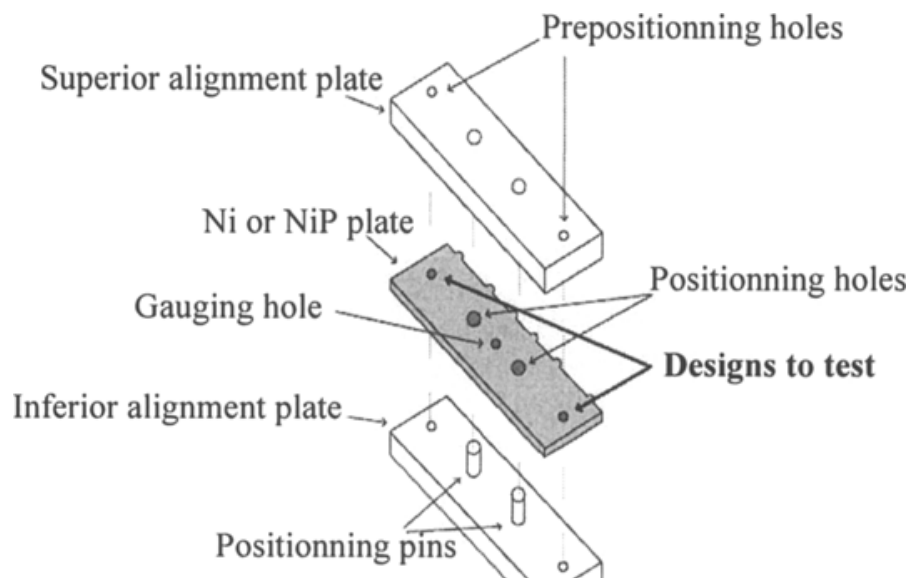

Fig. 9.

Device for the determination of the maximal press-fitting force 
The shaft enters in the prepositioning hole. We align the axis of the press with the axis of the shaft and we process to the press-fitting (strictly speaking).

In the test for the determination of the resistive torque associated with each design, we process it differently. Figure 10 presents the system:

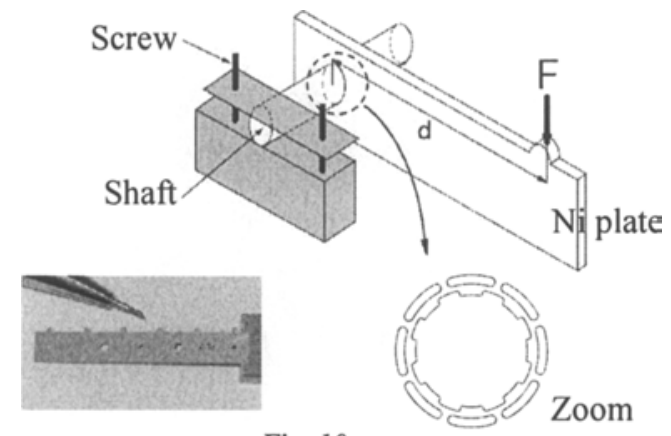

Fig. 10.

Device for the determination of the resistive torque

With the press, we come in contact with the plate at a distance $d$ from the shaft. Thus by knowing the force necessary to make the plate turn and the distance $d$, we manage to obtain the resistive torque associated with the design tested.

\section{Results}

Due to the different parameters we would like to analyse, we made the experiments on seven different designs:

- $\quad 8$ fluting, with flexion, $r=1 / 2$

- $\quad 8$ fluting, no flexion, $r=1 / 2$

- 24 fluting, no flexion, $r=3 / 4$

- $\quad 3$ fluting, with flexion, $r=3 / 4$

- $\quad 3$ fluting, no flexion, 15 channels, $r=3 / 4$

- $\quad 3$ fluting, with flexion, $r=1 / 4$

- $\quad 3$ fluting, no flexion, $r=1 / 4$

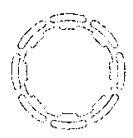

NB: $r=$ ratio between design surface and shaft surface 
As the production of samples by LIGA process takes quite a long time, we do not have many points of measurement. Nevertheless, we managed to obtain the global trend for each design. We specify that each point on the following graphs represents one measure.

\subsection{Press fitting force}

Experimental conditions: temperature: $21^{\circ} \mathrm{C} \pm 2^{\circ} \mathrm{C}$, press-fitting speed: $1 \mathrm{~mm} / \mathrm{s}, \mathrm{L}=$ $0.4 \mathrm{~mm}, \mathrm{E}=155 \mathrm{GPa}, \mu=0.2, \mathrm{D}=994 \mu \mathrm{m} \pm 0.5$ et $\mathrm{i}=2-27 \mu \mathrm{m}$

Figure 11 presents the results of the press-fitting test:

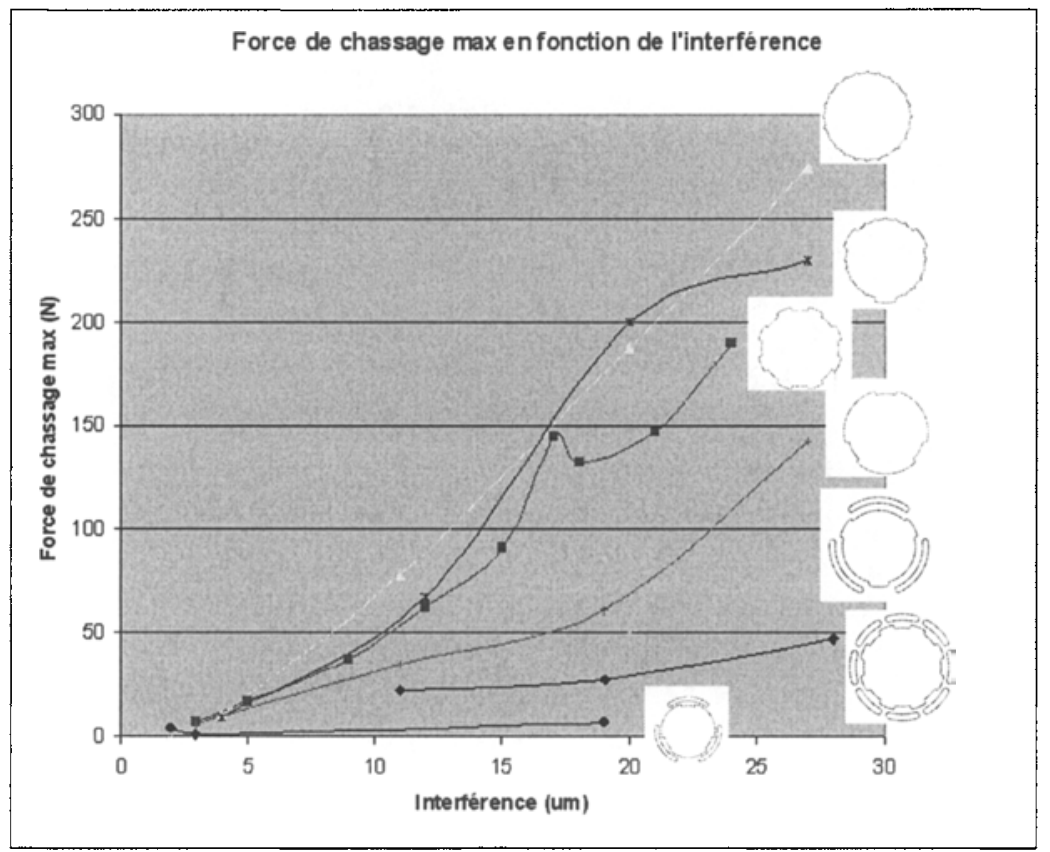

Fig. 11 .

Evolution of the maximal press-fitting force with the interference

\subsubsection{Results analysis}

The goal here was to observe the maximal press-fitting force value for each design and for a range of interference from 2 to $27 \mu \mathrm{m}$.

As we expected, designs with flexion oblongs reach a lower value than the others, which means that the matter is less constrained and probably does not reach the plastic field. This can be interesting for reversible applications or for devices which require a very high precision in axial positioning. 
Conversely, designs from the top of the graph require a bigger force to obtain the same results, which means that the contact will be stronger but the precision in the axial positioning will probably be more uncertain.

\subsection{Resistive torque}

The experimental conditions remain the same and we chose a distance $\mathrm{d}$ equal to 5 $\mathrm{mm}$. Fig. 12 presents the results of the resistive torque test:

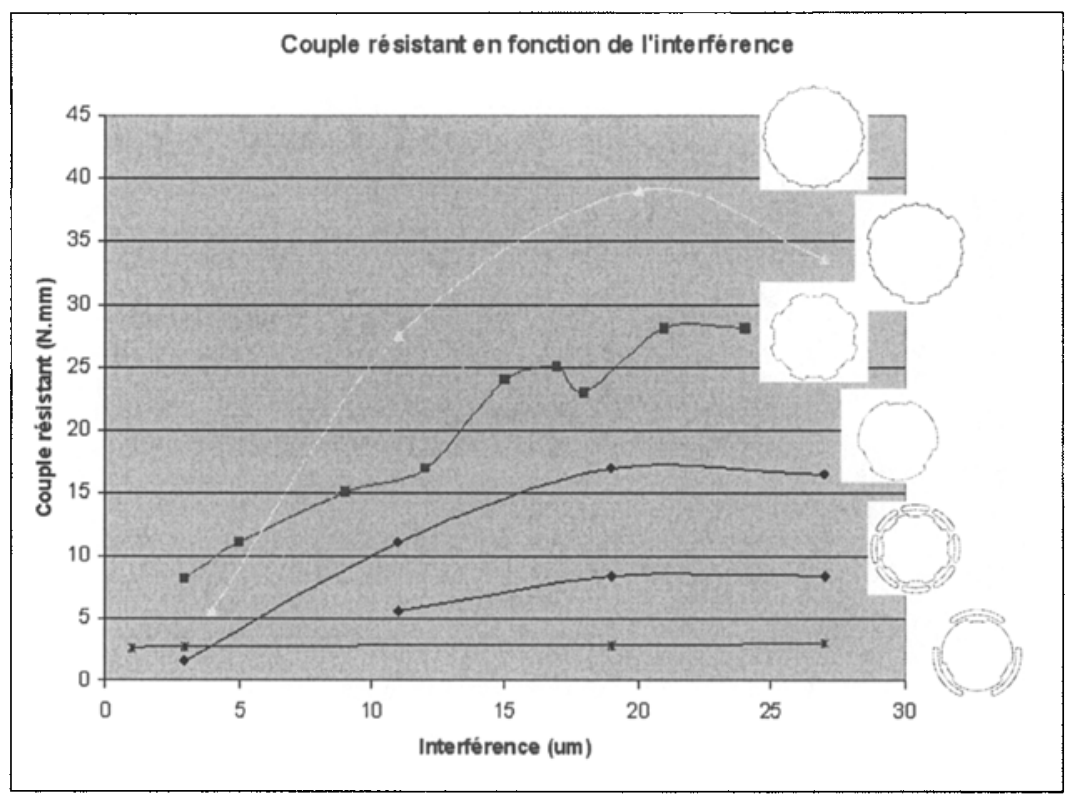

Fig. 12.

Evolution of the resistive torque with the interference

\subsubsection{Results analysis}

We notice here the designs with the higher $r$ ratio have a better resistive torque that the others, which seems logical as the torque directly depends on the contact surface between the shaft and the receptor. Moreover, we observe a kind of saturation for each design over an interference of about $20 \mu \mathrm{m}$. This could be explained by the fact that the matter has reached a certain deformation (plastic or not) and the surface in contact remains fairly constant despite the increase in the tightening. Nevertheless, this phenomenon has to be confirmed by other tests.

NB: We do not have results for the design with 3 fluting, with flexion, $r=3 / 4$ because it did not even manage to support the manipulation to be tested. We can sim- 
ply ensure that its comportment is very bad and its performance may be inferior to the worst case tested.

\section{Perspectives}

Despite the very few designs we have already tested, some guidelines appeared to us. First of all, the flexion oblongs play a great part in the stiffness of the contact. For the same design, the maximal of the press-fitting force is divided by four due to these empty spaces. Secondly, the proportion of the receptor surface also plays a great role. When we pass from a $r$ ratio of $1 / 4$ to $1 / 2$, we increase the resistive torque from about $33 \%$, and it is almost the same from $1 / 2$ to $3 / 4$.

Another approach which could be a lead for such a study would be the influence of the material. It could be interesting to build a table which would gather these kinds of values for the main materials used in industry. In particular we think of silicon which has a lot of particularities that could be very promising in this field of study. However, to begin to press-fit in silicon, we first have to determine a design which will reduce drastically the value of the maximum press-fitting force in order not to start a crack in the monocrystal of this material. The shape with 8 fluting, with flexion, $r=1 / 2$ would seem to be interesting for this application but it still has to be proved.

To determine these designs, we based our reflection on common sense, the fact that flexion is stiffer than traction/compression and that contact surface ratio would not be necessary equal to one. Yet we could also ask a finite elements software to give us its own optimised shape, given the right boundary conditions application. Thus, we would be able to compare what is theoretically calculated by a soft, with the shape we have chosen intuitively. The next step would be to adapt the form given by the computer to obtain something feasible, and to test this design to measure its performance. With such an approach, we would have a solid basis to work on, especially if the values obtained in concrete terms match with the results given by the simulation.

\section{References}

1. E. Fortini, Dimensioning for Interchangeable Manufacture, Industrial Press (1967)

2. F. Bourgeois, Vers la Maîtrise de la Qualité des Assemblages de Précision (EPFL, Lausanne, 2007) 